\title{
Analysis of the two accelerator concepts foreseen for the neutral beam injector of the International Thermonuclear Experimental Reactor
}

\author{
G. Fubiani* \\ Université de Toulouse; UPS, INPT; LAPLACE (Laboratoire Plasma et Conversion d'Energie); 118 route de Narbonne, \\ F-31062 Toulouse cedex 9, France \\ and CNRS; LAPLACE; F-31062 Toulouse, France \\ R. S. Hemsworth \\ ITER Joint Work Site, CEA Cadarache, F-13108, St. Paul lez Durance, France
}

H. P. L. de Esch and L. Svensson

DSM/DRFC, Association EURATOM-CEA, CEA Cadarache, F-13108, St. Paul lez Durance, France

(Received 10 February 2009; published 20 May 2009)

\begin{abstract}
Typical high-energy negative ion electrostatic accelerators such as the ones designed for fusion applications produce a significant amount of secondary particles. These particles may originate from coextracted electrons, which flow from the ion source, impacting the accelerator grids or as by-products of collisions between accelerated negative ions and the residual background gas, in the accelerator. Secondary emission particles may carry a non-negligible power and consequently must be precisely studied. The electrostatic-accelerator-Monte-Carlo-simulation code (EAMCC) [G. Fubiani et al., Phys. Rev. ST Accel. Beams 11, 014202 (2008)] was developed in order to provide a three-dimensional characterization of power and current deposition on all parts of the accelerator. The code includes all the relevant physics associated with secondary emission processes and consequently may be used as a tool for design improvement. In this paper, the two accelerator designs considered for the International Thermonuclear Experimental Reactor, that is, the multiaperture-multigrid and the single gap single aperture (SINGAP) designs, are discussed and their predicted performances compared. Simulations have been compared with measurements on prototype accelerators of the SINGAP type. Reasonable agreement between EAMCC calculations and measurements of backstreaming ions and transmitted electrons was found.
\end{abstract}

\section{INTRODUCTION}

Plasma heating and current drive requirements for the International Thermonuclear Experimental Reactor (ITER) include the use of high power neutral beam (NB) injectors. Each of the ITER NB injectors must deliver about $1 \mathrm{MeV}, 17 \mathrm{~A}$ (equivalent) of neutral deuterium atoms (i.e., $17 \mathrm{MW}$ ) to the fusion plasma [1,2]. The injector is composed of an ion source producing a high negative deuterium ion current density (typically of the order of $28 \mathrm{~mA} / \mathrm{cm}^{2}$ ), an electrostatic accelerator delivering $40 \mathrm{~A}$ of negative ion beamlets at $1 \mathrm{MeV}$ (40 MW of power), a neutralizer which converts parts of the beam into highenergy neutrals (neutralization efficiency of $\simeq 60 \%$ ) [3], and a residual ion dump.

One of the main drawbacks of the negative ion electrostatic accelerators for high power NB injectors is the significant amount of secondary particles which are produced inside the accelerator [4]. Secondary particles originate from collisions between the accelerated negative ions and the residual background gas and from the collisions between particles (primary or secondary) and material

*gwenael.fubiani@laplace.univ-tlse.fr surfaces in the accelerator. The former includes both loss of negative ions by stripping, ionization of the gas and the production of electrons by those processes. In addition, the negative ion plasma source will produce electrons that are coextracted with the negative ions. All these unwanted particles will absorb a significant amount of power from the power supplies which may be deposited further downstream the accelerator due to impacts of the particles with the grids and their support structures.

This paper will focus on the two accelerator concepts being developed for ITER, the multiaperture-multigrid (MAMuG) [2,5] and the single gap single aperture (SINGAP) [6] accelerators. A schematic view of the two accelerators is shown in Fig. 1. Both accelerators consist of a plasma grid (PG) which separates the ion source from the accelerator, an extraction grid (EG), and a series of acceleration grids (AGs). The extraction grid is necessary in order to both compensate space charge defocusing of the negative ion beamlets and collect unwanted coextracted electrons from the plasma source. The major difference between the two accelerator concepts is on the AG design. The MAMuG accelerator is a five-stage acceleration system where each grid (including the PG and EG) is divided into 16 groups of 80 apertures (1280 holes in total). The 
(a)

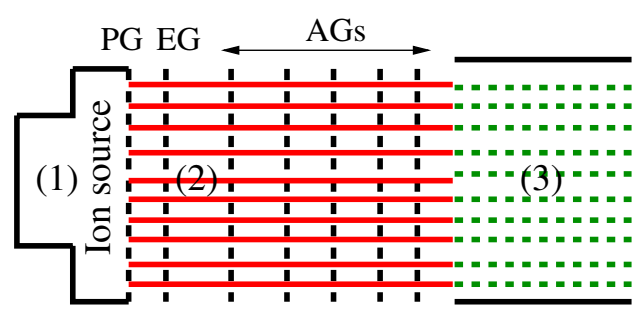

(b)

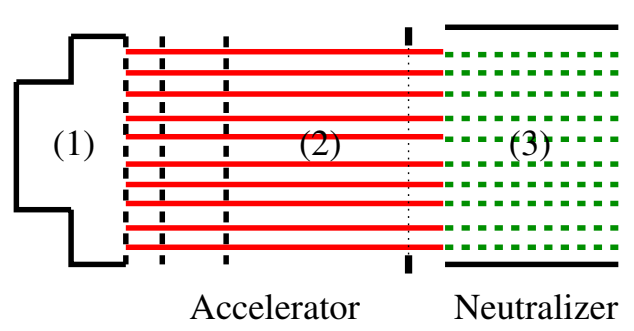

FIG. 1. (Color) Schematic representation of a negative ion electrostatic accelerator. Neighboring components of the neutral beam injector are also shown for clarity. In region (1), the negative ion source; region (2), the high-energy electrostatic accelerator. There are currently two concepts foreseen for ITER; the multiaperture-multigrid accelerator (MAMuG) [upper plot, (a)], which utilizes five acceleration grids (AG) to accelerate negative ions to an energy of $1 \mathrm{MeV}$, and the single gap single aperture (SINGAP) [lower plot, (b)] concept consisting uniquely of two AGs. The last AG for the MAMuG accelerator is similar to the other AGs, i.e., having 1280 apertures, while for SINGAP it has 16 large rectangular apertures, one per group of 80 beamlets. In region (3), the neutralizer is shown; the highenergy negative ion beamlets are gradually neutralized (green dashed lines). Neutralization efficiency is around $60 \%$.

entire aperture array is rectangular, $(577 \times 1535) \mathrm{mm}^{2}$. The potential difference between two AGs is $\simeq 200 \mathrm{kV}$. Concerning SINGAP, the main acceleration region corresponds to a single gap, i.e., two AGs. The PG, EG, and first $\mathrm{AG}$ are similar to the ones of the MAMuG accelerator, that is, with 1280 apertures in 16 groups of 80 apertures, while the second (and last) AG, which is at ground potential, has 16 large rectangular apertures, and the 80 beamlets from one group of apertures in the first AG pass through one of the 16 apertures. The accelerating potential across the last (main) acceleration stage is $945 \mathrm{kV}$ for SINGAP. As there are more grids in the MAMuG concept, secondary particles have a higher probability of impact in that accelerator than in the SINGAP accelerator, while in the latter the secondary particles are likely to be transmitted through the large apertures in the grounded grid. These considerations have important implications for both accelerator concepts.

While the ITER-MAMuG accelerator has been simulated in a previous work [4], the SINGAP concept still needed to be theoretically investigated. The paper is organized as follows: Sec. II gives an overview of the physics involved in secondary particle production and reviews the features of the electrostatic-accelerator-Monte-Carlosimulation code [4] (EAMCC). EAMCC was specifically de- veloped to model secondary emission processes inside high-energy electrostatic accelerators. In Sec. III, a detailed calculation of the SINGAP accelerator is performed together with a direct comparison with the features of the MAMuG concept. In Sec. IV, a calculation of the currents to be supplied by the power supplies is shown for the two accelerators.

\section{MODELING OF SECONDARY PARTICLE PRODUCTION INSIDE A NEGATIVE ION ACCELERATOR}

Secondary particle production will be responsible for significant parasitic power absorption from the power supplies and induce a large power deposition on the accelerator and on beam line components with the high-energy, high current accelerators foreseen for ITER. Unwanted particles originate from several sources: (i) coextracted electrons from the negative ion source, (ii) secondary electron production from particle impacts (electrons, heavy ions, and neutrals) on accelerator grids, (iii) collisions between the accelerated negative ions and the residual background gas inside the accelerator (stripping of one or two electrons from the $\mathrm{D}^{-}$and ionization of the background gas). Coextracted electrons are mostly collected by the EG due to embedded permanent magnets deflecting them onto the grid. Typical extraction voltage for the future ITER device is about $9 \mathrm{kV}$ and, assuming about one electron per extracted ion $[7,8]$, the corresponding total extracted electron current is of the order of 50 A [4]. Consequently, power deposition from coextracted electrons on the ITER-EG will be large, that is $\simeq 450 \mathrm{~kW}$. Background gas pressure is also significant inside the accelerator; the residual gas originates from both the plasma source (typically operated at a $\mathrm{D}_{2}$ gas filling pressure of $0.3 \mathrm{~Pa}$ ) and from the neutralizer area. Interaction between the gas and negative ions is calculated to result in a stripping loss for the latter ranging from $\simeq 23 \%$ to $29 \%$ depending on the accelerator type. Ionization of the background gas causes production of positive ions amounting to about $\simeq 4 \%$ to $8 \%$ of the total accelerated negative ion current transmitted toward the neutralizer. Each secondary charged particle (positive ions and electrons) produced is accelerated and deflected by the electric and magnetic fields inside the accelerator and may induce more secondaries after an impact with the accelerator grids. This chain of reactions is responsible for a non-negligible heat load on the grids (of the order of a few MW [4]) and must be understood in detail.

The EAMCC code [4] was developed in order to simulate secondary particle effects together with negative ion beamlets transport inside the ITER accelerator. The physics included in the code is general and can be applied to any type of accelerator. EAMCC is a relativistic particle tracking code. The trajectory of a particle is calculated inside prescribed electrostatic and magnetic fields; the latter 
being calculated from external codes. EAMCC calculates the trajectories of all particles, including secondary particles. Collisions are described using a Monte-Carlo method; the type of collisions considered in the code are (i) electron and heavy ion/neutral collisions with accelerator grids, (ii) negative ion single and double stripping reactions, and (iii) ionization of background gas by negative ions and neutrals. The first version of EAMCC is fully three dimensional (3D) for the magnetic field and twodimensional (2D) cylindrical symmetric for the electric field. Negative ion transport is reduced to two beamlets, which is necessary in order to account for the asymmetry between two neighboring apertures arising from the magnetic field from the embedded permanent magnets inside the EG. Simulation of the full accelerator, that is including the 1280 apertures for ITER, is obtained by scaling the two-beamlets calculation. The code has recently been upgraded to cope with 3D electric potential maps. This modification is necessary in order to simulate accelerators with a noncylindrical symmetric geometry, such as the SINGAP concept. The new version of the code, EAMCC$3 \mathrm{D}$, can include the whole accelerator geometry in the calculation. Both algorithms allow the user to calculate 3D power deposition from secondary particles on accelerator grids (and consequently find high power density regions), compute negative ion stripping ratio, transmitted power per species (both toward the ion source and the neutralizer), and, among others, describe secondary particle production versus beam optics.

\section{THE SINGAP ACCELERATOR}

As explained previously, the SINGAP concept [6] accelerates negative deuterium ions to high energy in several intermediate steps. The three first grids (PG, EG, and the first acceleration grid, called pre-AG in the SINGAP context) are similar to the ones of MAMuG, that have aperture arrays consisting of 16 groups of 80 apertures (total 1280 holes) with a transverse cross section of $0.89 \mathrm{~m}^{2}$. The EG voltage is set at $9.6 \mathrm{kV}$ and the pre- $\mathrm{AG}$ at $55 \mathrm{kV}$; the final beam acceleration to $1 \mathrm{MeV}$ is performed in one step. Each group of beamlets from each group of 80 apertures then passes through one of the 16 large apertures in the final (ground potential) electrode. The filling pressure in the ion source is $0.3 \mathrm{~Pa}$ (with no source operation and the system at room temperature).

Figure 2 shows the postacceleration gap for ITERSINGAP; for clarity the plots shows only five beamlets from two of the groups of apertures together with secondary particles. The square blocks on the left and right side of Fig. 2 are thick metallic structures bolted on the pre-AG and grounded grid (GG) edge [6]; these so-called "kerbs" steer the beams horizontally and help counteract the space charge repulsion between the beamlets within each group. In addition, the GG is slightly V-shaped in the vertical direction (with the tip of the "V" to the upstream side).

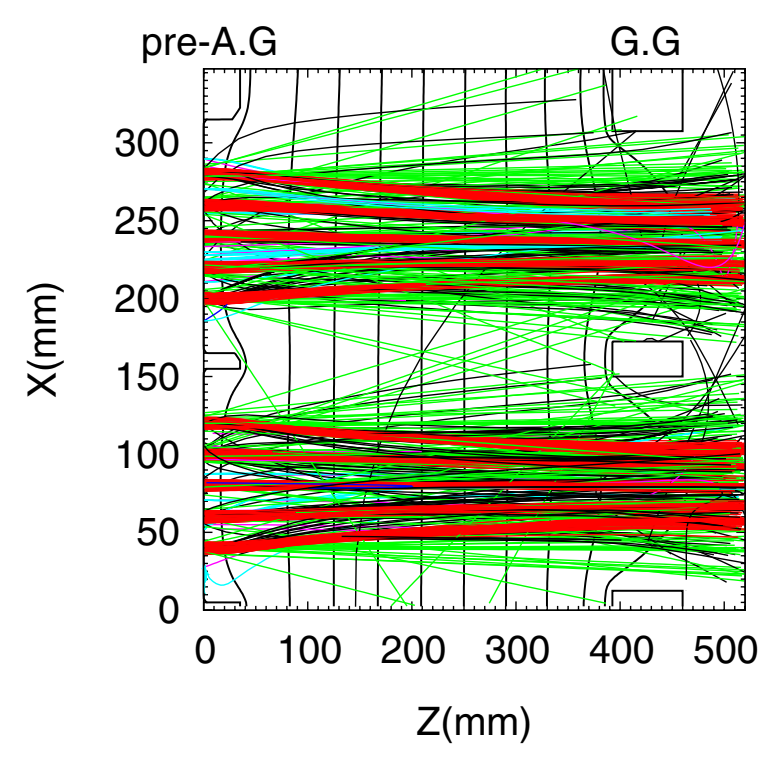

FIG. 2. (Color) Geometry of the SINGAP negative ion based electrostatic accelerator. The plot shows five beamlets from two aperture groups over the postacceleration gap. From left to right: preacceleration grid (AG) and grounded grid (GG) (total acceleration voltage of $945 \mathrm{kV}$ in the postacceleration gap). Primary and secondary particles are shown; negative deuterium ions (red color), neutrals $\left(\mathrm{D}^{0}\right)$ (green), positive deuterium ions $\left(\mathrm{D}^{+}\right)$(dark blue), positive deuterium molecular ions $\left(\mathrm{D}_{2}^{+}\right)$(purple), electrons (black), and lastly electrons produced by positive ion impacts on the back side of the preacceleration grid (light blue).

The resulting electric field profile in the acceleration gap creates the required vertical steering of the beam groups. Because of the large openings on the GG electrode of the SINGAP accelerator, most particles produced inside the postacceleration gap are transmitted through the grounded grid towards the neutralizer (the neutralizer entrance is located at the right side of the plot; it is not shown). The total power carried toward this region of the injector is high because of the large potential difference between the pre$\mathrm{AG}$ and the GG (945 kV for the ITER-SINGAP design). Furthermore, a significant amount of positive ions (which originate from either double stripping of negative ions or ionization of the background gas) will hit the back of the pre-AG grid. These backstreaming positive ions will produce secondary electrons, which are accelerated towards the grounded grid. Most reach a final energy of $\simeq 945 \mathrm{keV}$ as interception inside the postacceleration gap is only with residual gas molecules. They consequently pass through the large apertures in the grounded grid, towards the neutralizer.

\section{A. Secondary particle power deposition estimated by EAMCC}

Table I shows a summary of the results of calculations using the EAMCC code for the power deposited on the accelerator grids and transmitted (toward the neutralizer 
TABLE I. Total power generated by secondary particles for MAMuG and SINGAP accelerators calculated by the EAMCC code. The total accelerated $\mathrm{D}^{-}$power is $40 \mathrm{MW}$. $P_{\text {grid }}$ corresponds to the total power deposited on grids, $P_{\text {neut }}$ power transmitted toward the neutralizer, and $P_{\text {src }}$ back into the negative ion source. The numbers shown include contribution from (i) stripping reactions, (ii) ionization of background gas, (iii) electron production from particle impacts on accelerator grids, and (iv) coextracted plasma electrons.

\begin{tabular}{ccccc}
\hline \hline & Species & $P_{\text {grid }}(\mathrm{MW})$ & $P_{\text {neut }}(\mathrm{MW})$ & $P_{\text {src }}(\mathrm{MW})$ \\
\hline MAMuG & $\mathrm{e}^{-}$ & 7 & 0.6 & None \\
& $\mathrm{D}^{0}$ & 0.1 & 2.2 & None \\
& $\mathrm{D}^{+}$ & Negligible & Negligible & 0.14 \\
& $\mathrm{D}_{2}^{+}$ & 0.13 & None & 0.74 \\
SINGAP & $\mathrm{e}^{-}$ & 1.7 & 8 & None \\
& $\mathrm{D}^{0}$ & Negligible & 1.7 & None \\
& $\mathrm{D}^{+}$ & 0.04 & 0.05 & 0.03 \\
& $\mathrm{D}_{2}^{+}$ & 0.28 & None & 0.36 \\
\hline \hline
\end{tabular}

and the negative ion source) by secondary particles for the two accelerators foreseen for ITER, MAMuG, and SINGAP. For MAMuG, the EG voltage is set at $9.4 \mathrm{kV}$ and each of the five AGs have a potential difference of $200 \mathrm{kV}$; all grids have 1280 apertures. Estimates for the ITER-MAMuG accelerator have already been published elsewhere [4]. Characteristics of the power deposition profile from secondary particles are widely different between the two devices. In both cases, most power is carried by secondary electrons. In ITER-SINGAP, electrons absorb a total of $9.7 \mathrm{MW}$ of power from the generators which is mostly transmitted toward the neutralizer (8 MW, 10.7 A); the rest is deposited on the grids. A detailed study of the origins of these electrons shows that 1.4 MW (1.5 A) is carried by particles which were produced as by-products of positive ion impacts on the back side of the pre-AG grid (facing the neutralizer), while about 1.2 MW (1.2 A) are from coextracted electrons. It was assumed that $52 \mathrm{~A}$ of electrons were coextracted with the negative ions (one electron per extracted negative ion) corresponding to a current density of $26.5 \mathrm{~mA} / \mathrm{cm}^{2}$. Consequently, only $1.9 \%$ of the electrons extracted from the ion source are accelerated to full energy. The remaining electron power (5.4 MW, $8 \mathrm{~A}$ ) is produced inside the postacceleration gap.

These estimates do not include positive ions which will be extracted from the plasma formed by beam ionization of the background gas downstream of the grounded grid. It has been calculated that about 0.4 A [9] may be extracted from that plasma into the postacceleration region. Many of these ions will hit the back of the pre-AG at an energy of $945 \mathrm{keV}$ and consequently produce secondary electrons. The secondary electron emission coefficient is of the order of three electrons per impact at normal incidence and significantly larger for impacts inside the pre-AG apertures (at glancing incidence) [4]). A rather conservative estimate for the electron power originating from these ions (neglecting ion impacts inside apertures) is $1.1 \mathrm{MW}(1.2 \mathrm{~A})$, and that power mostly exits the grounded grid and is transmitted towards the neutralizer.

For SINGAP, the total negative ion stripping was found to be of the order of $23 \%$ (the stripping profile is shown in Fig. 3); 1.7 MW of power is transmitted as neutrals towards the neutralizer, and $390 \mathrm{~kW}$ as positive ions (mostly molecular deuterium ions) flows back towards the ion source. The latter, though not as high as will be the case with the ITER-MAMuG accelerator (880 $\mathrm{kW} \mathrm{[4]),} \mathrm{positive} \mathrm{ion}$ impacts will cause erosion of the back plate of the plasma source by sputtering. Footprints left by positive ion beamlet impacts have been observed experimentally inside the ion source of high-energy NB injectors and are similar to those foreseen for ITER [10].

The ITER-MAMuG concept has quite a different power density deposition profile compared to ITER-SINGAP. In the case of the MAMuG, most electron power is deposited on the accelerator grids ( $7 \mathrm{MW}$ ) with a significantly lower amount transmitted toward the neutralizer $(\simeq 820 \mathrm{~kW})$. This major difference is simply due to the higher impact probability on the accelerator grids compared to SINGAP because of the low transparency of the acceleration grids in MAMuG. Furthermore, total negative ion stripping ratio is $29 \%$ (see Fig. 3) which is larger than for the ITERSINGAP concept due to a typically higher background

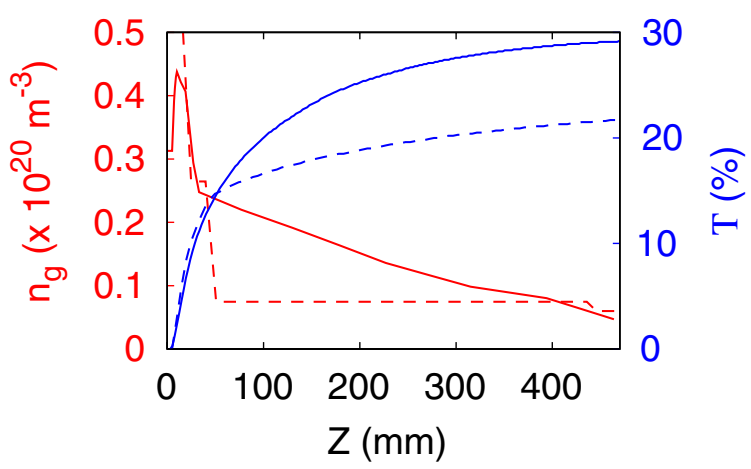

FIG. 3. (Color) Background gas density profile $n_{g}(z)$ (left axis, red color) and negative deuterium ion stripping rate $\Gamma(z)$ (right axis, blue color) as a function of propagation distance inside the accelerator is shown for both the MAMuG accelerator (solid lines) and the SINGAP accelerator (dashed lines). The gas density for MAMuG is calculated using a Monte-Carlo method described in Ref. [11] while, for SINGAP, using a classical conductance approach [19]. A filling pressure of $0.3 \mathrm{~Pa}$ in the ion source is assumed (with no source operation and the system at room temperature). For SINGAP the accelerator pressure was assumed to be $0.03 \mathrm{~Pa}$, for MAMuG it follows from the MC calculations in Ref. [11]. In addition, a source gas temperature $T_{g}=2000 \mathrm{~K}$ during discharge operation was assumed for MAMuG and $300 \mathrm{~K}$ for SINGAP. This difference in assumptions matters for the extractor $(Z<20 \mathrm{~mm}$ ) but is of no consequence in the postaccelerator as the gas will have accommodated to $300 \mathrm{~K}$ both for SINGAP and for MAMuG. 
gas pressure inside the accelerator $[4,11]$. Consequently, this imposes a higher required negative ion current density to be extracted from the ion source in order to have $40 \mathrm{~A}$ of $1 \mathrm{MeV}$ ions accelerated out of the accelerator; $28.5 \mathrm{~mA} / \mathrm{cm}^{2}$ for MAMuG compared to $26.5 \mathrm{~mA} / \mathrm{cm}^{2}$ in SINGAP.

Last, the total parasitic power carried by secondary particles (obtained by summing both power deposited on accelerator grids and transmitted) is similar for the two accelerator concepts; $10.9 \mathrm{MW}$ for ITER-MAMuG and 12.1 MW for ITER-SINGAP. In both cases this is a large fraction, $27 \%$ to $30 \%$, of the total negative ion power leaving the accelerator (40 MW).

\section{B. Experimental measurements}

In this section, we compare EAMCC calculations with experimental data. The first experiment simulated was performed in the SINGAP-prototype at the CEA (French Atomic Energy Commission) laboratory in Cadarache, France. The Cadarache $1 \mathrm{MV}$ negative ion beam facility is capable of accelerating $100 \mathrm{~mA}$ of negative hydrogen or deuterium ions up to $1 \mathrm{MeV}$. The negative ions are first accelerated in the preaccelerator to energies of $10-50 \mathrm{keV}$ and thereafter up to maximum energy in a single stage postaccelerator [12]. An experimental campaign was dedicated to the measurement of positive ion power deposition of the back side of the negative ion source. Most positive ions produced inside the accelerator are believed to reach the ion source. In order to verify this hypothesis, helium gas was added inside the accelerator in order to enhance positive ion production rates. The experiment was carried with one beamlet only extracted from the PG through a $14 \mathrm{~mm}$ hole aperture. Positive ion power deposited on a copper target located at the rear of the ion source was determined from the temperature rise of the target as measured by a thermocouple buried therein. The most relevant collision processes between deuterium ions, neutrals, and helium gas were added to EAMCC $[13,14]$; a summary of the reactions included is shown in Table II. Figure 4(a) shows the ratio of positive ion beam power with respect to the negative ion power collected on the calo-

TABLE II. Processes involved in the destruction of negative ions and ionization of helium gas which are included in EAMCC $[13,14]$.

\begin{tabular}{lcc}
\hline \hline Reaction \# & Process & Label \\
\hline 1 & $\mathrm{D}^{-}+\mathrm{He} \rightarrow \mathrm{D}^{0}+\mathrm{He}+\mathrm{e}^{-}$ & Sgl strip. neg. ion \\
2 & $\mathrm{D}^{0}+\mathrm{He} \rightarrow \mathrm{D}^{+}+\mathrm{He}+\mathrm{e}^{-}$ & Sgl strip. neut. \\
3 & $\mathrm{D}^{-}+\mathrm{He} \rightarrow \mathrm{D}^{+}+\mathrm{He}+2 \mathrm{e}^{-}$ & Double stripping \\
4 & $\mathrm{D}^{-}+\mathrm{He} \rightarrow \mathrm{D}^{-}+\mathrm{He}^{+}+\mathrm{e}^{-}$ & Ionization \\
5 & $\mathrm{D}^{0}+\mathrm{He} \rightarrow \mathrm{D}^{0}+\mathrm{He}^{+}+\mathrm{e}^{-}$ & Ionization \\
6 & $\mathrm{D}^{-}+\mathrm{He} \rightarrow \mathrm{D}^{0}+\mathrm{He}^{+}+2 \mathrm{e}^{-}$ & Ioniz. \& strip. \\
7 & $\mathrm{D}^{0}+\mathrm{He} \rightarrow \mathrm{D}^{+}+\mathrm{He}^{+}+2 \mathrm{e}^{-}$ & Ioniz. \& strip. \\
\hline \hline
\end{tabular}
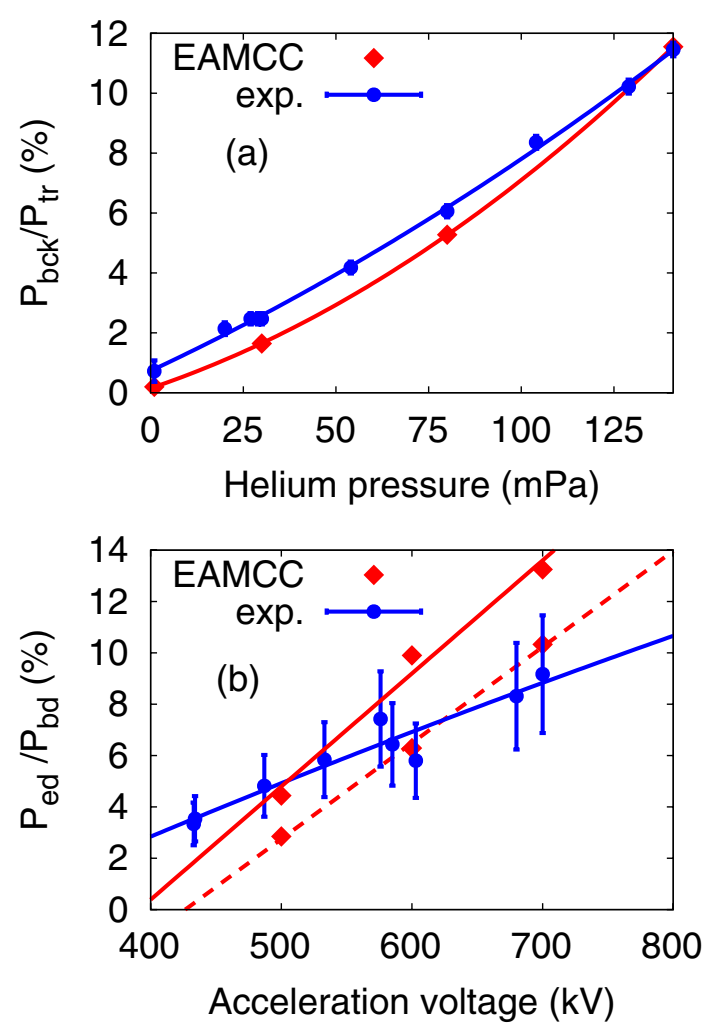

FIG. 4. (Color) Comparison between experimental data and EAMCC. In (a), experiments conducted at the CEA laboratory in Cadarache, France on a SINGAP accelerator. Positive ion beam power measured on the back side of the ion source is shown normalized to the negative ion beam power measured downstream of the accelerator on a calorimeter versus background helium gas pressure (which was used for this series of experiments in order to enhance positive ion production). Experimental data (blue color) and simulations (red color) are reported. In (b), SINGAP experiment at JAEA in Naka, Japan. Ratio of power collected on an electron dump and the negative ion beam dump is shown versus acceleration voltage. Lines (solid and dashed) correspond to first order polynomial fits.

rimeter downstream the accelerator as a function of background helium pressure for both experimental data (blue color) and EAMCC calculations (red color). A good agreement between measurements and simulations is found. For large helium pressures, typically $P_{\mathrm{He}} \gtrsim 0.1 \mathrm{~Pa}$, plasma effects inside the accelerator may be non-negligible. This will induce errors in EAMCC estimates.

A series of experiments have also been conducted at the megavolt test facility at the Japanese Atomic Energy Agency (JAEA) in Naka, Japan [15] on an ITER-like SINGAP accelerator [15]. For practical reasons the first stage AG of the prototype MAMuG accelerator and the first stage of the MAMuG power supply had to be used for the SINGAP preaccelerator. Therefore the pre-AG potential was $1 / 5$ of the total acceleration voltage. A total of 15 beamlets were accelerated. After acceleration the ions drifted towards a beam dump located $\simeq 3 \mathrm{~m}$ downstream 
of the grounded grid. The electrons were deflected onto two electron dumps; only one of which was equipped with a thermocouple for temperature measurement and subsequent determination of the power to the target from the measured temperature rise. The power carried by the electrons transmitted out of the accelerator was measured, and this supports the estimates from the calculations discussed in Sec. III A. Figure 4(b) shows the ratio of power measured on the electron dump divided by the power collected on the beam dump for both experimental data (blue color) and EAMCC calculations (red color). The agreement between simulation and experiments is reasonable. Experimental error bars were calculated based on data fluctuations. Unfortunately, there is uncertainty in the electron dump position as the latter is known to have moved during the experimental campaign. The data shown in red, solid line, represents the ratio of the power on the second electron dump for the designed electron dump locations and the dashed line is for a hypothetical location $1 \mathrm{~cm}$ closer to the negative ion beam path. Because of the large distance between the last AG and the beam dumps, a small variation in the electron dump position would have caused a significant change in the power collected.

Differences between simulations and experiments may originate from different sources. The main difficulty in EAMCC is to correctly estimate the plasma meniscus shape. Field maps calculated by the code SLACCAD are used. SLACCAD [16] does not perform any plasma physics calculations. Consequently, the plasma meniscus is calculated rather simply by imposing a vanishing electrostatic field inside the negative ion source. Another important source of error may be the appearance of a beamlet halo. Power density profiles of negative ion beamlets measured experimentally show that a part of the beamlet has divergence that is high compared to the bulk of the beamlet [17], which is commonly referred to as the beamlet "halo." The optics of the main part of the beamlet and the secondary particle production in the accelerator can be affected by the presence of the divergent ions making up such a halo in the accelerator. Last, error bars on cross sections (which are sometimes quite large) for the physical chemistry of deuterium inside the accelerator is an additional uncertainty which must be considered.

Based on the results discussed in this section, that is, the large electron production observed experimentally for SINGAP accelerators together with the calculations reported here for the reference ITER-SINGAP design (see Table I) have led to the selection of the MAMuG concept for ITER.

\section{ITER ACCELERATOR POWER SUPPLY CHARACTERISTICS}

Required characteristics of the power supplies for the ITER-MAMuG accelerator have been calculated using EAMCC. The parameters used are from the reference design which is a $9 \mathrm{kV}$ extraction grid together with five acceleration grids, each at a potential difference of $200 \mathrm{kV}$, and a final negative ion energy of $1009 \mathrm{kV}$. We have included a beamlet halo and the following hypothetical model of the halo has been used to simulate its effect on the negative ion beam optics and secondary particle production. Cesium (Cs) is injected into the ion source to lower the work function of metal surface [18] and consequently enhance the negative ion yield from surface production. The injected Cs will cover the inner surfaces of the ion source and migrate from the ion source side of the plasma grid across the surface into the accelerator, and some Cs will enter the accelerator as vapor. Some of the Cs entering/migrating into the accelerator will cover to some degree the downstream surface of the plasma grid. Of the $\mathrm{D}^{0}$ atoms flowing out of the source, some will be reflected off surfaces and hit the downstream side of the PG. Some of the $\mathrm{D}^{0}$ impinging in an annulus around each of the 1280 apertures will be backscattered as negative ions and be accelerated through the subsequent grids, forming a divergent halo around each beamlet. In calculating the currents that the power supplies for the ITER-MAMuG accelerator have to deliver, the current density from the annuli on the downstream side of the plasma grid is adjusted so that $\mathrm{a} \simeq 14 \%$ beamlet halo appears in the total accelerated current of $\simeq 40 \mathrm{~A}$; consequently, only about $34 \mathrm{~A}$ of negative ions emerging from the accelerator have a good divergence, as assumed for the ITER neutral beam injector design.

A summary of the required power supply capabilities for ITER-MAMuG is shown in Fig. 5. The plot displays the total currents flowing through each gap inside the accelerator (linked to an independent power supply) together with the associated power required to be delivered by the generators. These power estimates represent absolute minima in order to provide the necessary energy to accelerate $40 \mathrm{~A}$ of negative ions to $1 \mathrm{MeV}$. Total secondary particle production is superior to the case calculated in Table I owing to the addition of a beamlet halo. Total parasitic power absorption is found to be 13.4 MW (2.5 MW in excess mostly caused by direct hitting of negative ions within the halo with the accelerator grids). Total power deposited on the accelerator grids amounts to $9.6 \mathrm{MW}$ (red color in Fig. 5) while power transmitted toward the neutralizer is of the order of $3 \mathrm{MW}$ (mostly carried by neutrals) and $\simeq 840 \mathrm{~kW}$ back inside the plasma source (exclusively from positive ions). Note that secondary electron power transmitted towards the neutralizer is significant, $\simeq 820 \mathrm{~kW}$.

Calculation of the ITER-SINGAP power supply characteristics is shown in Fig. 6 using the same parameters introduced in Sec. III. This simulation includes a $15 \%$ halo ratio to the total $40 \mathrm{~A}$ of accelerated negative ion current at the exit of the accelerator. Note that most of the negative ions within the halo are transmitted outside the accelerator. Consequently, the power supply characteristics 


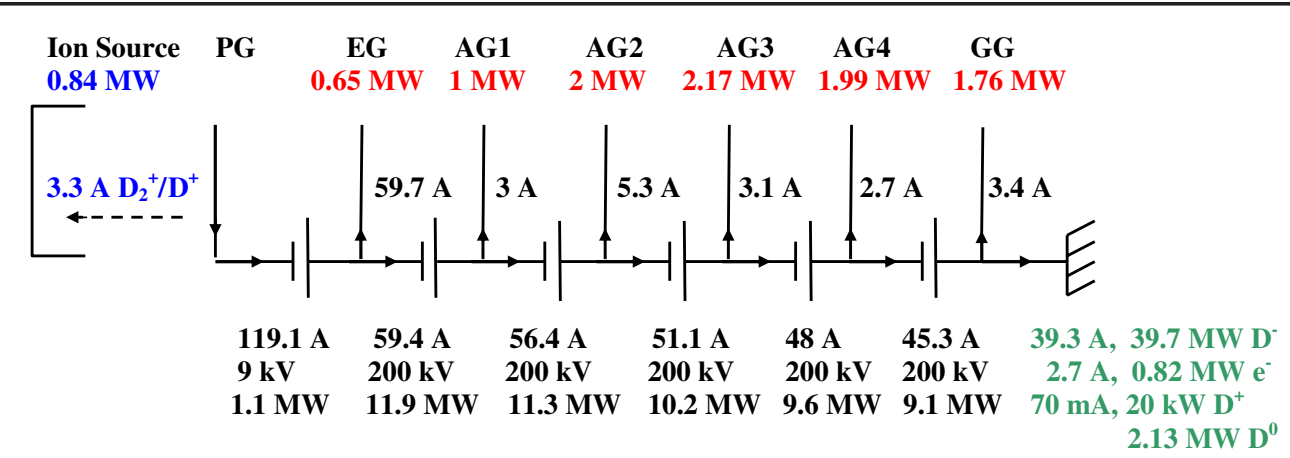

FIG. 5. (Color) ITER-MAMuG power supply characteristics. The schematic shows the powers and corresponding currents flowing through the power supplies across each gap (bottom), generated by both negative ions and secondary particles. In dark green (bottom right), powers and currents corresponding to particles transmitted toward the neutralizer are shown. In red (top) are the impact powers from secondary particles on grids. In blue (left), power and current transmitted toward the ion source (carried by positive ions). The calculation assumed $\simeq 40 \mathrm{~A}$ of accelerated negative ions at the exit of the accelerator (total power of $\simeq 40 \mathrm{MW}$ ) including $\simeq 14 \%$ of halo current, i.e., there are $34 \mathrm{~A}$ of negative ions extracted from the ion source and 5.4 A produced on the back of the plasma grid. The total power to be supplied to the accelerator is $53.2 \mathrm{MW}$; $13.4 \mathrm{MW}$ of which is simply parasitic power absorbed by secondary particles.

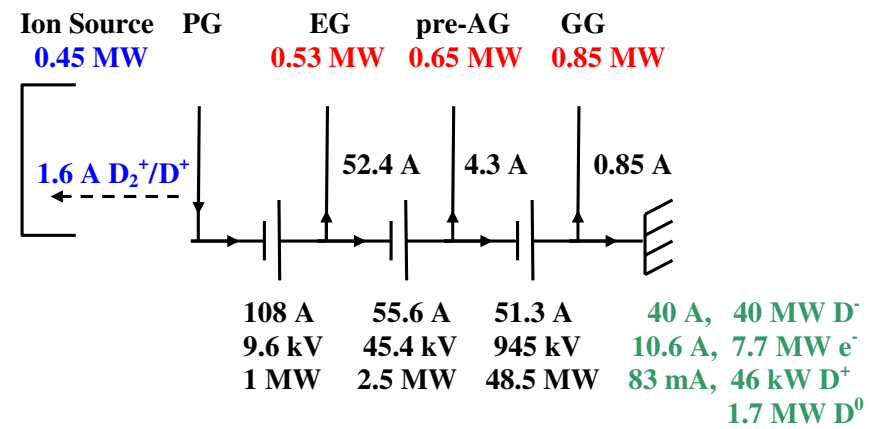

FIG. 6. (Color) ITER-SINGAP power supply characteristics. Shown in dark green (bottom right), are the powers and currents corresponding to particles transmitted towards the neutralizer. In red (top) are the impact powers from secondary particles on grids. In blue (left), power and current transmitted toward the ion source. The calculation assumed $40 \mathrm{~A}$ of accelerated negative ions at the exit of the accelerator including $15 \%$ of halo current. Total power generated by the power supplies amount to $52 \mathrm{MW}$ (12 MW is parasitic power absorbed by secondary particles).

are very similar with or without halo. Total power generated by the power supplies is slightly lower than the reference design ITER-MAMuG concept, corresponding to a parasitic power absorption from secondary particles of 12 MW (which represents $30 \%$ of the negative deuterium ion beamlet power at the exit of the accelerator). Total secondary particle power transmitted toward the neutralizer amounts to $9.5 \mathrm{MW}$ which is substantially higher than the MAMuG accelerator ( $\simeq 3 \mathrm{MW})$.

\section{CONCLUSION}

In this paper, we have calculated the effects of secondary particle production inside the two electrostatic accelerator concepts being developed for the ITER high-energy NB injectors. The relativistic Monte-Carlo particle tracking code EAMCC was modified in order to cope with arbitrary geometries; previous versions were suited uniquely for 2D cylindrical symmetric devices. It is found that the two accelerator concepts for ITER, MAMuG, and SINGAP have a significantly different power deposition profile induced by secondary particles inside the NB injector. In both cases, a high parasitic power absorption from the power supplies is calculated: of the order of $13.5 \mathrm{MW}$ for ITER-MAMuG and $12 \mathrm{MW}$ for ITER-SINGAP. In ITERMAMuG most power is deposited inside the accelerator (9.6 MW on grids) while a lower amount is transmitted toward the neutralizer and the negative ion source (total of 3.8 MW). For ITER-SINGAP, the power on grids is reduced, $\simeq 2 \mathrm{MW}$ since a significant part of the power carried by the secondary particles is transmitted through the accelerator (total $\simeq 10 \mathrm{MW}$ ). This difference in the fraction of secondary particles transmitted as opposed to being intercepted inside the accelerator for the two accelerator concepts is a direct consequence of the geometry of the accelerators, MAMuG having several, sequential, small apertures through which each beamlets must pass compared to the completely open main acceleration stage and very large apertures in the grounded grid of SINGAP. The high electron fraction transmitted downstream of a SINGAP accelerator has also been observed experimentally in an ITER-like prototype. Based on the calculations presented here and those experimental results, the MAMuG concept was chosen for ITER. One of the critical issues that remain to be solved in ITER-MAMuG is the handling of the high power density deposited by positive ions on the back plate of the negative ion source. Based on calculations, it is found that the peak power density may exceed $2 \mathrm{~kW} / \mathrm{cm}^{2}$.

\section{ACKNOWLEDGMENTS}

The authors acknowledge useful discussions with A. Simonin. This work was performed in the framework 
of a collaboration between the CNRS-LAPLACE (Laboratoire Plasma et Conversion d'Energie) Laboratory and CEA (French Atomic Energy Commission), Cadarache, France.

[1] ITER Physics Basis Editors, ITER Physics Expert Group Chairs and Co-Chairs, ITER Joint Central Team and Physics Integration Unit, and ITER EDA, Nucl. Fusion 39, 2137 (1999).

[2] T. Inoue et al., Fusion Eng. Des. 56-57, 517 (2001).

[3] R. S. Hemsworth and T. Inoue, IEEE Trans. Plasma Sci. 33, 1799 (2005).

[4] G. Fubiani, H. de Esch, A. Simonin, and R. Hemsworth, Phys. Rev. ST Accel. Beams 11, 014202 (2008).

[5] K. Watanabe, Y. Fujiwara, M. Hanada, M. Kashiwagi, T. Kitagawa, K. Miyamoto, T. Morishita, Y. Okumura, T. Takayanagi, and M. Taniguchi, Rev. Sci. Instrum. 71, 1231 (2000).

[6] H. de Esch, R. Hemsworth, and P. Massmann, Fusion Eng. Des. 73, 329 (2005).

[7] R. Trainham, C. Jacquot, D. Riz, A. Simonin, K. Miyamoto, Y. Fujiwara, and Y. Okumura, Rev. Sci. Instrum. 69, 926 (1998).

[8] E. Speth et al., Nucl. Fusion 46, S220 (2006).

[9] A. Lifschitz, T. Minea, G. Maynard, K. Katsonis, J.
Bretagne, and A. Simonin, Nucl. Fusion (to be published).

[10] M. Taniguchi, T. Inoue, N. Umeda, M. Kashiwagi, K. Watanabe, H. Tobari, M. Dairaku, and K. Sakamoto, Rev. Sci. Instrum. 79, 02 C110 (2008).

[11] A. Krylov and R. S. Hemsworth, Fusion Eng. Des. 81, 2239 (2006).

[12] L. Svensson, D. Boilson, H. de Esch, R. Hemsworth, and A. Krylov, Nucl. Fusion 46, S369 (2006).

[13] C.F. Barnett, Oak Ridge National Laboratory Technical Report No. ORNL-6086, 1990.

[14] R. D. DuBois and A. Kövèr, Phys. Rev. A 40, 3605 (1989).

[15] H. de Esch, L. Svensson, T. Inoue, M. Taniguchi, N. Umeda, M. Kashiwagi, and G. Fubiani, Fusion Eng. Des. (to be published).

[16] J. Pamela, Rev. Sci. Instrum. 62, 1163 (1991).

[17] T. Inoue, M. Kashiwagi, M. Taniguchi, M. Dairaku, M. Hanada, K. Watanabe, and K. Sakamoto, Nucl. Fusion 46, S379 (2006).

[18] W. G. Graham, in Proceedings of the Second International Symposium on the Production and Neutralization of Negative Hydrogen Ions and Beams, edited by $\mathrm{T}$. Sluyters (Brookhaven National Laboratory, Upton, New York, 1980), p. 126.

[19] S. Dushman and J. M. Lafferty, Scientific Foundations of Vacuum Technique (John Wiley \& Sons Inc., New York, 1962). 